\title{
Bottomonium-like states at Belle
}

\section{Pavel Krokovny*}

Budker Institute of Nuclear Physics and Novosibirsk State University

E-mail: krokovny@inp.nsk.su

We report the first observation of $\Upsilon(5 S) \rightarrow \Upsilon(1,2,3 S) \pi^{0} \pi^{0}$ decays. The neutral partner of the $Z_{b}^{ \pm}(10610)$, the $Z_{b}^{0}(10610)$ decaying to $\Upsilon(2,3 S) \pi^{0}$, is observed for the first time with a $6.5 \sigma$ significance using a Dalitz analysis of $\Upsilon(5 S) \rightarrow \Upsilon(2,3 S) \pi^{0} \pi^{0}$ decays. First results on the analysis of the three-body $\Upsilon(5 S) \rightarrow\left[B \bar{B}^{*}+\text { c.c. }\right]^{ \pm} \pi^{\mp}$ and $\Upsilon(5 S) \rightarrow\left[B^{*} \bar{B}^{*}\right]^{ \pm} \pi^{\mp}$ including first observation of $Z_{b}^{ \pm}(10610) \rightarrow\left[B \bar{B}^{*}+\text { c.c. }\right]^{ \pm}$and $Z_{b}^{ \pm}(10650) \rightarrow\left[B^{*} \bar{B}^{*}\right]^{ \pm}$are also reported. The results are obtained with a $121.4 \mathrm{fb}^{-1}$ data sample collected with the Belle detector at the $\Upsilon(5 S)$ resonance at the KEKB asymmetric-energy $e^{+} e^{-}$collider.

The XV International Conference on Hadron Spectroscopy,

November 4 - 8, 2013

Nara, Japan

${ }^{*}$ Speaker. 

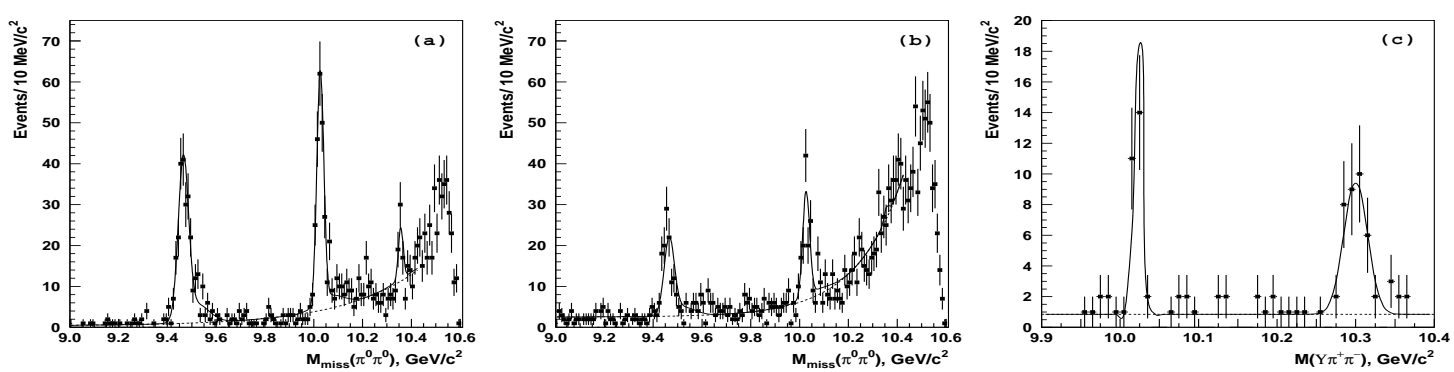

Figure 1: The $\pi^{0} \pi^{0}$ missing mass distribution for $\Upsilon(n S) \pi^{0} \pi^{0}$, (a) $\Upsilon(n S) \rightarrow \mu^{+} \mu^{-}$and (b) $\Upsilon(n S) \rightarrow e^{+} e^{-}$ candidates. The $M\left(\Upsilon(1 S) \pi^{+} \pi^{-}\right)$distribution for $\Upsilon(2 S) \rightarrow \Upsilon(1 S) \pi^{+} \pi^{-}$candidates is shown in (c). Points with error bars represent the data. The solid curves show the fit result while the dashed curves correspond to the background contributions.

Table 1: Signal yield, MC efficiency, measured branching fraction, number of selected events and purity.

\begin{tabular}{lccccc}
\hline \hline Final state & Signal yield & $\varepsilon, \%$ & $\mathscr{B}, 10^{-3}$ & Events & Purity \\
\hline$\Upsilon(1 S) \rightarrow \mu^{+} \mu^{-}$ & $261 \pm 15$ & 11.2 & $2.28 \pm 0.13$ & 247 & 0.95 \\
$\Upsilon(1 S) \rightarrow e^{+} e^{-}$ & $123 \pm 13$ & 5.61 & $2.15 \pm 0.23$ & 140 & 0.78 \\
$\Upsilon(2 S) \rightarrow \mu^{+} \mu^{-}$ & $241 \pm 18$ & 8.04 & $3.77 \pm 0.28$ & 253 & 0.87 \\
$\Upsilon(2 S) \rightarrow e^{+} e^{-}$ & $108 \pm 13$ & 3.58 & $3.84 \pm 0.46$ & 151 & 0.66 \\
$\Upsilon(2 S) \rightarrow \Upsilon(1 S) \pi^{+} \pi^{-}$ & $24 \pm 5$ & 2.27 & $2.85 \pm 0.60$ & 28 & 0.86 \\
$\Upsilon(3 S) \rightarrow \mu^{+} \mu^{-}$ & $49 \pm 12$ & 2.60 & $0.71 \pm 0.17$ & 114 & 0.43 \\
$\Upsilon(3 S) \rightarrow e^{+} e^{-}$ & $9 \pm 14$ & 1.19 & $0.29 \pm 0.44$ & not used & - \\
\hline \hline
\end{tabular}

\section{1. $\Upsilon(5 S) \rightarrow \Upsilon(n S) \pi^{0} \pi^{0}$ decays}

We reconstruct $\Upsilon(n S)$ candidates from pairs of leptons $\left(e^{+} e^{-}\right.$and $\left.\mu^{+} \mu^{-}\right)$. An additional decay channel is used for the $\Upsilon(2 S): \Upsilon(2 S) \rightarrow \Upsilon(1 S)\left[l^{+} l^{-}\right] \pi^{+} \pi^{-}$. Muon and electron candidates are required to be positively identified. Candidate $\pi^{0}$ mesons are selected from pairs of photons with an invariant mass within $15 \mathrm{MeV} / \mathrm{c}^{2}$ of the nominal $\pi^{0}$ mass. $\Upsilon(5 S) \rightarrow \Upsilon(n S)\left[l^{+} l^{-}\right] \pi^{0} \pi^{0}$ candidates are identified via the missing mass recoiling against the $\pi^{0} \pi^{0}$ system, $M_{\text {miss }}\left(\pi^{0} \pi^{0}\right)$. More details can be found in Ref. [1]. Figure 1 shows the extraction of the $\Upsilon(n S)$ signal yield. Results are summarized in Table 1. The cross section is calculated from $\sigma=\frac{N_{\text {sig }}}{\varepsilon \mathscr{B}(\mathrm{Y}(n S) \rightarrow X) \mathscr{L}\left(1+\delta_{\text {ISR }}\right)}$, where $N_{\text {sig }}$ is the number of signal events, $\varepsilon$ is the reconstruction efficiency, $\mathscr{B}(\Upsilon(n S) \rightarrow X)$ is the branching fraction of the $\Upsilon(n S)$ to the reconstructed final state $X, \mathscr{L}$ is the integrated luminosity and $\left(1+\delta_{\text {ISR }}\right)=0.666 \pm 0.013$ is the initial state radiation (ISR) correction factor. Weighted averages are found to be $\sigma\left(e^{+} e^{-} \rightarrow \Upsilon(5 S) \rightarrow \Upsilon(1 S) \pi^{0} \pi^{0}\right)=(1.16 \pm 0.06 \pm 0.10) \mathrm{pb}, \sigma\left(e^{+} e^{-} \rightarrow \Upsilon(5 S) \rightarrow\right.$ $\left.\Upsilon(2 S) \pi^{0} \pi^{0}\right)=(1.87 \pm 0.11 \pm 0.23) \mathrm{pb}$, and $\sigma\left(e^{+} e^{-} \rightarrow \Upsilon(5 S) \rightarrow \Upsilon(3 S) \pi^{0} \pi^{0}\right)=(0.98 \pm 0.24 \pm$ $0.19) \mathrm{pb}$.

The amplitude analysis of the three-body $\Upsilon(5 S) \rightarrow \Upsilon(n S) \pi^{0} \pi^{0}$ decays utilizes an unbinned maximum likelihood fit. We parameterize the three-body decay amplitude as a sum of quasi-twobody amplitudes: $M\left(s_{1}, s_{2}\right)=A_{Z 1}+A_{Z 2}+A_{f_{0}}+A_{f_{2}}+A_{\mathrm{nr}}$, where $A_{Z 1}$ and $A_{Z 2}$ are amplitudes for contributions from the $Z_{b}^{0}(10610)$ and $Z_{b}^{0}(10650)$, respectively; the amplitudes $A_{f_{0}}, A_{f_{2}}$ and $A_{\mathrm{nr}}$ 

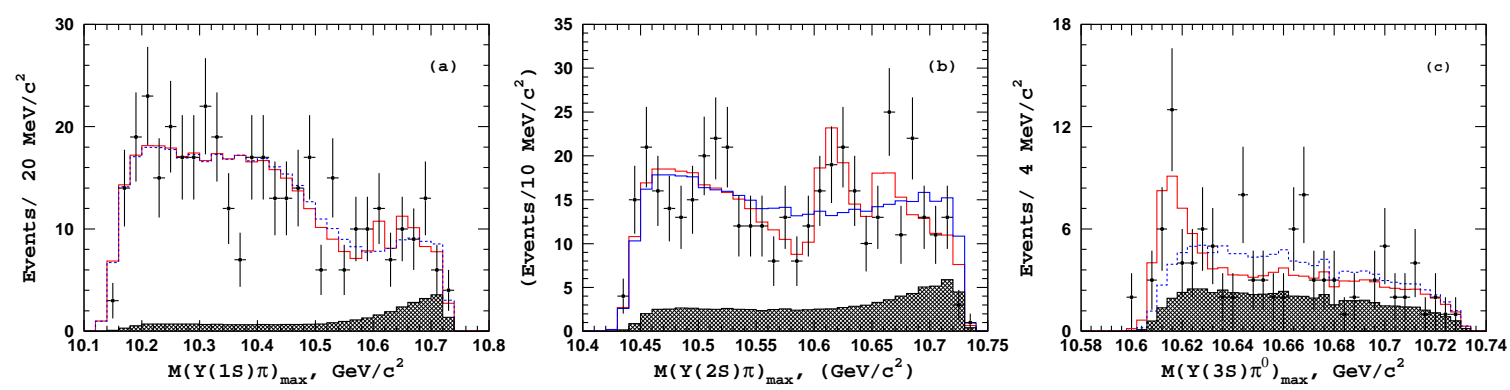

Figure 2: Comparison of the fit results (open histograms) with experimental data (points with error bars) for the $\Upsilon(n S) \pi^{0} \pi^{0}$ events in the signal region. Red and blue open histograms show the fit with and without $Z_{b}^{0}$ 's, respectively. Hatched histograms show the background components.

are the contributions from the $\pi^{0} \pi^{0}$ system in an $f_{0}(980), f_{2}(1275)$ and a non-resonant state, respectively. The masses and widths of $Z_{b}$ resonances are fixed at the values obtained from the $\Upsilon(n S) \pi^{+} \pi^{-}$analysis: $M\left(Z_{1}\right)=10607.2 \mathrm{MeV} / \mathrm{c}^{2}, \Gamma\left(Z_{1}\right)=18.4 \mathrm{MeV} / \mathrm{c}, M\left(Z_{2}\right)=10652.2 \mathrm{MeV} / \mathrm{c}^{2}$, $\Gamma\left(Z_{2}\right)=11.5 \mathrm{MeV} / \mathrm{c}$ [2]. We use a Flatté function for the $f_{0}(980)$ and a Breit-Wigner function for the $f_{2}(1275)$. The non-resonant amplitude $A_{\mathrm{nr}}$ is parameterized as $A_{\mathrm{nr}}=A_{\mathrm{nr}}^{1} e^{i \phi_{\mathrm{nr}}^{1}}+A_{\mathrm{nr}}^{2} e^{i \phi_{\mathrm{nr}}^{2}} s_{3}$. As the fit is sensitive only to the relative amplitudes and phases between decay modes, we fix $A_{\mathrm{nr}}^{1}=10.0$ and $\phi_{\mathrm{nr}}^{1}=0.0$. The logarithmic likelihood function is defined as $\mathscr{L}=-2 \sum \log \left\{\varepsilon\left(s_{1}, s_{2}\right) f_{\mathrm{sig}} S\left(s_{1}, s_{2}\right)+\right.$ $\left.\left(1-f_{\text {sig }}\right) B\left(s_{1}, s_{2}\right)\right\}$, where $S\left(s_{1}, s_{2}\right)$ denotes $\left|M\left(s_{1}, s_{2}\right)\right|^{2}$ convoluted with the detector resolution function, $\varepsilon\left(s_{1}, s_{2}\right)$ describes variation of the reconstruction efficiency over the Dalitz plot and $f_{\text {sig }}$ is the fraction of signal events in the data sample. The fraction $f_{\text {sig }}$ is determined separately for each $\Upsilon(n S)$ decay mode (see Table 1). The function $B\left(s_{1}, s_{2}\right)$ describes the distribution of background events over the phase space. Both $\varepsilon\left(s_{1}, s_{2}\right) \cdot S\left(s_{1}, s_{2}\right)$ and $B\left(s_{1}, s_{2}\right)$ are normalized to unity. Results of the fits are shown in Fig. 2 (b) and (c) as one-dimensional projections that look similar to the corresponding distributions for the $\Upsilon(n S) \pi^{+} \pi^{-}$decays [2]. A $Z_{b}^{0}$ signal is most clearly seen in $M\left(\Upsilon \pi^{0}\right)_{\max }$. The values and errors of amplitudes and phases obtained from the fit are presented in [1]. The statistical significance of the $Z_{b}^{0}(10610)$ signal in the $\Upsilon(2 S) \pi^{0} \pi^{0}$ sample is $5.3 \sigma$. In addition, the $Z_{b}^{0}(10610)$ statistical significance is $4.7 \sigma$ in the fit to the $\Upsilon(3 S) \pi^{0} \pi^{0}$ sample. The signal for the $Z_{b}^{0}(10610)$ is not significant in the fit to the $\Upsilon(1 S) \pi^{0} \pi^{0}$ events due to the smaller relative branching fraction. The signal of the $Z_{b}^{0}(10650)$ is not significant in either $\Upsilon(1 S) \pi^{0} \pi^{0}$ or $\Upsilon(2 S) \pi^{0} \pi^{0}$ datasets. We perform a simultaneous fit of the $\Upsilon(2 S) \pi^{0} \pi^{0}$ and $\Upsilon(3 S) \pi^{0} \pi^{0}$ data samples. No constraints between samples are imposed on signal model parameters and the background description. The combined significance of the $Z_{b}^{0}(10610)$ signal is $6.8 \sigma$.

We study possible uncertainties due to parameterization of the background PDF, variation of signal efficiency over the Dalitz plot and detector resolution function. The model uncertainty is estimated using various description of $S$-wave contribution. The significance of the $Z_{b}^{0}(10610)$ signal exceeds $6.5 \sigma$ in all cases. We use this value as the final value for the $Z_{b}^{0}(10610)$ significance.

\section{2. $\Upsilon(10860) \rightarrow B^{(*)} \bar{B}^{*} \pi$ Decays}

$B$ decays are reconstructed in the following channels: $B^{+} \rightarrow J / \psi K^{+}, B^{+} \rightarrow \overline{D^{0}} \pi^{+}, B^{0} \rightarrow$ $J / \psi K^{* 0}, B^{0} \rightarrow D^{(*)-} \pi^{+}$. We identify $B$ candidates by their invariant mass $M(B)$ and momentum 

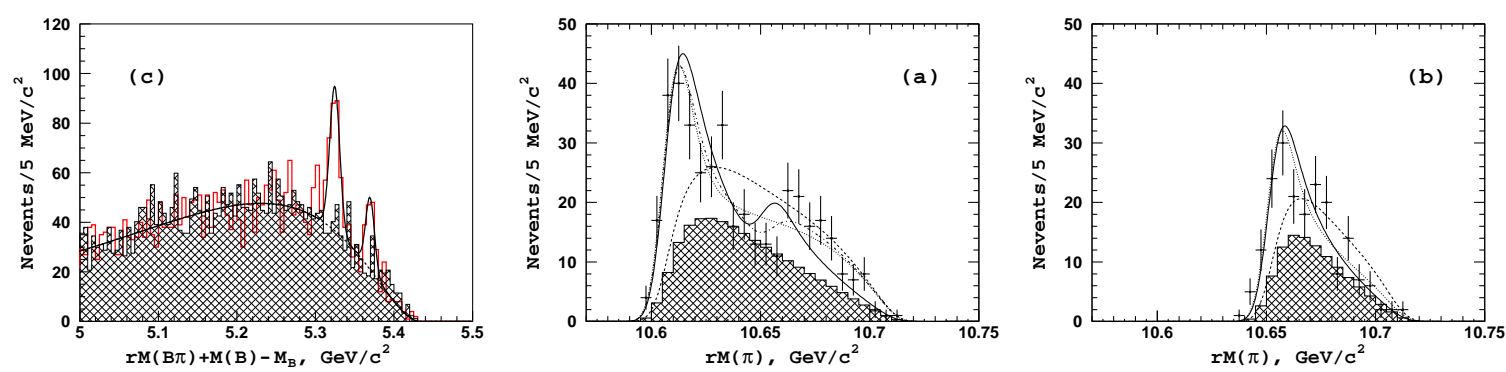

Figure 3: (a) $M_{r}(B \pi)$ distributions for selected $B$ candidates in data. Hatched histogram shows distribution for events in the $M(B)$ sidebands. (b) $M_{r}(\pi)$ distribution for right-sign $B \pi$ combinations for $\Upsilon(10860) \rightarrow$ $B B^{*} \pi$ and (c) $\Upsilon(10860) \rightarrow B^{*} B^{*} \pi$ candidate events. Points with error bars are data, the solid line is the result of the fit with the nominal model, the dashed line - fit to pure non-resonant amplitude, the dotted line - fit to a single $Z_{b}$ state plus a non-resonant amplitude, and the dash-dotted - two $Z_{b}$ states and a non-resonant amplitude. The hatched histogram represents background component.

$P(B)$ in the c.m. We require $M(B)$ to be within 30 to $40 \mathrm{MeV} / c^{2}$ (depending on the $B$ decay mode) of the nominal $B$ mass. Reconstructed $B^{+}$or $B^{0}$ candidates are then combined with a $\pi^{-}$candidate and a recoil mass to the $B \pi$ combination, $M_{r}(B \pi)$, is calculated as $M_{r}(B \pi)=\sqrt{E_{\mathrm{cms}}^{2}-P_{B \pi}^{2}}$, where $E_{\mathrm{cms}}$ is the c.m. energy and $P_{B \pi}$ is the measured three-momentum of the $B \pi$ combination. More details can be found in Ref. [3]. The $M_{r}(B \pi)+M(B)-M_{B}$ distribution for the data is shown in Fig. 3(a), where clear peaks are visible in the $B B^{*} \pi$ and $B^{*} B^{*} \pi$ signal regions. The fit to this distribution gives $N_{B B \pi}=1 \pm 14, N_{B B^{*} \pi}=184 \pm 19$ and $N_{B^{*} B^{*} \pi}=82 \pm 11$ signal events. The statistical significance of the observed $B B^{*} \pi$ and $B^{*} B^{*} \pi$ signal is $9.3 \sigma$ and $5.7 \sigma$, respectively. For the subsequent analysis of the internal structures of the three-body decays, we require $\mid\left(M_{r}(B)+\right.$ $\left.M(B)-M_{B}\right)-M_{B^{*}} \mid<0.015 \mathrm{GeV} / c^{2}$ to select $\Upsilon(10860) \rightarrow B B^{*} \pi$ events and $\mid\left(M_{r}(B)+M(B)-\right.$ $\left.M_{B}\right)-\left(M_{B^{*}}+E_{\gamma}\right) \mid<0.015 \mathrm{GeV} / c^{2}$, where $E_{\gamma}=0.049 \mathrm{GeV}$, to select $\Upsilon(10860) \rightarrow B^{*} B^{*} \pi$ events. For selected $B^{(*)} B^{(*)} \pi$ candidate events, we calculate the mass recoiling against the charged pion: $M_{r}(\pi)=\sqrt{E_{\mathrm{cms}}^{2}-P_{\pi}^{2}}$, where $P_{B \pi}$ is the measured three-momentum of the charged pion.

The $M_{r}(\pi)$ distributions for right-sign $B \pi$ combinations in the $B B^{*} \pi$ and $B^{*} B^{*} \pi$ signal regions are shown in Fig. 3. Excesses of signal events over the expected background levels at lower mass edges of the $M_{r}(\pi)$ spectra are clearly visible for both final states. The distribution of signal $\Upsilon(10860) \rightarrow B B^{*} \pi$ events is parameterized with the following model $S_{B B^{*} \pi}(m)=\left(A_{Z_{b}(10610)}+\right.$ $\left.A_{N R}\right) \times E_{B B^{*} \pi}(m)$, where $A_{N R}$ is the non-resonant amplitude parameterized as a complex constant and the $Z_{b}(10610)$ amplitude is a Breit-Wigner function. As a variation of this nominal model, we also add a second Breit-Wigner amplitude to account for possible $Z_{b}(10650) \rightarrow B B^{*} \pi$ decay. We also fit the data with only the $Z_{b}(10610)$ channel included in the decay amplitude. The results of these fits are shown in Fig. 3(b). Two models give about equally good description of the data: nominal model and a model with additional non-resonant amplitude. However, we select the former one as our nominal model since adding a non-resonant amplitude does not improve the fit quality that much. The worst fit to the data is provided by a model with just a non-resonant amplitude. From this analysis, we find that the significance of the $Z_{b}(10610) \rightarrow B B^{*}$ signal is exceeding the $8 \sigma$ level.

As the nominal model for the $\Upsilon(10860) \rightarrow B^{*} B^{*} \pi$ decay, we use the following parameter- 
Table 2: List of branching fractions for the $Z_{b}^{+}(10610)$ and $Z_{b}^{+}(10650)$ decays.

\begin{tabular}{lcccccc}
\hline \hline $\mathscr{B}, \%$ & $\Upsilon(1 S) \pi^{+}$ & $\Upsilon(2 S) \pi^{+}$ & $\Upsilon(3 S) \pi^{+}$ & $h_{b}(1 P) \pi^{+}$ & $h_{b}(2 P) \pi^{+}$ & $B^{(*)} \bar{B}^{*}$ \\
\hline$Z_{b}(10610)$ & $0.32 \pm 0.09$ & $4.38 \pm 1.21$ & $2.15 \pm 0.56$ & $2.81 \pm 1.10$ & $4.34 \pm 2.07$ & $86.0 \pm 3.6$ \\
$Z_{b}(10650)$ & $0.24 \pm 0.07$ & $2.40 \pm 0.63$ & $1.64 \pm 0.40$ & $7.43 \pm 2.70$ & $14.8 \pm 6.22$ & $73.4 \pm 7.0$ \\
\hline \hline
\end{tabular}

ization: $S_{B^{*} B^{*} \pi}(m)=\left(A_{Z_{b}(10650)}+A_{N R}\right) \mathrm{E}_{B^{*} B^{*} \pi}(m)$. We also fit the data without a non-resonant component and with a non-resonant amplitude alone. Results of the fits are shown in Fig. 3(c); numerical values are given in [3]. The best description of the $B^{*} B^{*} \pi$ data is achieved in a model with only the $Z_{b}(10650)$ amplitude included. The addition of a non-resonant amplitude does not provide any significant improvement of the fit quality. The fit with a non-resonant amplitude alone gives a much worse likelihood value. From this analysis, we determine the significance of the $Z_{b}(10650) \rightarrow B^{*} B^{*}$ signal to be $6.8 \sigma$. In all fits discussed above, the masses and widths of the $Z_{b}$ states were fixed at the values obtained from the analysis of the $\Upsilon(n S) \pi^{+} \pi^{-}$and $h_{b}(m P) \pi^{+} \pi^{-}-$ final states [2].

\section{Conclusion}

We report the first observation of three-body $\Upsilon(5 S) \rightarrow \Upsilon(1,2,3 S) \pi^{0} \pi^{0}$ decays. The measured cross sections are $\sigma\left(e^{+} e^{-} \rightarrow \Upsilon(5 S) \rightarrow \Upsilon(1 S) \pi^{0} \pi^{0}\right)=(1.16 \pm 0.06 \pm 0.10) \mathrm{pb}, \sigma\left(e^{+} e^{-} \rightarrow \Upsilon(5 S) \rightarrow\right.$ $\left.\Upsilon(2 S) \pi^{0} \pi^{0}\right)=(1.87 \pm 0.11 \pm 0.23) \mathrm{pb}$, and $\sigma\left(e^{+} e^{-} \rightarrow \Upsilon(5 S) \rightarrow \Upsilon(3 S) \pi^{0} \pi^{0}\right)=(0.98 \pm 0.24 \pm$ $0.19) \mathrm{pb}$. The first observation of a neutral resonance decaying to $\Upsilon(2,3 S) \pi^{0}$, the $Z_{b}^{0}(10610)$, has been obtained in a Dalitz analysis of $\Upsilon(5 S) \rightarrow \Upsilon(2,3 S) \pi^{0} \pi^{0}$ decays. The statistical significance of the $Z_{b}^{0}(10610)$ signal is $6.5 \sigma$ including model and systematic uncertainties. Its measured mass, $M\left(Z_{b}^{0}(10610)\right)=10609 \pm 4 \pm 4 \mathrm{MeV} / \mathrm{c}^{2}$, is consistent with that measured in the analysis of $\Upsilon(5 S) \rightarrow \Upsilon(n S) \pi^{+} \pi^{-}$decays. The $Z_{b}^{0}(10650)$ signal is not significant in either $\Upsilon(1,2,3 S) \pi^{0} \pi^{0}$ channels. We also report measurement of branching fractions for three-body decays: $\mathscr{B}(\Upsilon(10860) \rightarrow$ $\left.\left[B \bar{B}^{*}+\text { c.c. }\right]^{+} \pi^{-}\right)=(28.3 \pm 2.9 \pm 4.6) \times 10^{-3}$ and $\mathscr{B}\left(\Upsilon(10860) \rightarrow\left[B^{*} \bar{B}^{*}\right]^{+} \pi^{-}\right)=(14.1 \pm 1.9 \pm$ $2.4) \times 10^{-3}$. For the $\Upsilon(10860) \rightarrow B \bar{B} \pi$ decay, we calculate a $90 \%$ confidence level upper limit of $\mathscr{B}\left(\Upsilon(10860) \rightarrow[B \bar{B}]^{+} \pi^{-}\right)<4.0 \times 10^{-3}$ (including systematic uncertainty). In addition, we report the ratio of the branching fractions $\frac{\mathscr{B}\left(Z_{b}(10610) \rightarrow B B^{*}\right)}{\sum_{n} \mathscr{B}\left(Z_{b}(10610) \rightarrow \mathrm{r}(n S) \pi\right), h_{b}(m P) \pi}=6.2 \pm 0.7 \pm 1.3_{-1.8}^{+0.0}$ and $\frac{\mathscr{B}\left(Z_{b}(10650) \rightarrow B^{*} B^{*}\right)}{\left.Z_{b}(10650) \rightarrow \mathrm{r}(n S) \pi, h_{b}(m P) \pi\right)}=2.8 \pm 0.4 \pm 0.6_{-0.4}^{+0.0}$. We calculate the relative fractions for $Z_{b}$ decays assuming that are saturated by the already observed $\Upsilon(n S)(n=1,2,3), h_{b}(m P)(m=1,2)$, and $B^{*} B^{(*)}$ channels. Combining results reported here with results on amplitude analysis from Ref. [3] one calculate relative fractions summarized in Table 2. All presented results are preliminary.

\section{References}

[1] P. Krokovny, A. Bondar et al., (Belle Collaboration), Phys. Rev. D 88, 052016 (2013).

[2] A. Bondar, A. Garmash, R. Mizuk et al. (Belle Collaboration), Phys. Rev. Lett. 108, 122001 (2012).

[3] I. Adachi et al., (Belle Collaboration), [arXiv:1209.6450]. 